\title{
The impact of photonuclear reaction models on propagation of ultrahigh energy cosmic rays
}

\author{
E. Kido, ${ }^{a, *}$ T. Inakura, ${ }^{b}$ M. Kimura, ${ }^{c}$ S. Nagataki, ${ }^{a}$ A. Tamii ${ }^{d}$ and Y. Utsuno ${ }^{e}$ \\ ${ }^{a}$ Astrophysical Big Bang Laboratory, RIKEN, \\ Wako, Saitama, Japan \\ ${ }^{b}$ Laboratory for Zero-Carbon Energy, Tokyo Instutute of Technology, \\ Meguro, Tokyo, Japan \\ ${ }^{c}$ Hokkaido University, \\ Sapporo, Hokkaido, Japan \\ ${ }^{d}$ Research Center for Nuclear Physics, Osaka University, \\ Ibaraki, Osaka, Japan \\ e Japan Atomic Energy Agency, \\ Tokai, Ibaraki, Japan \\ E-mail: eiji.kido@riken.jp, inakura@gmail.com, \\ masaaki@nucl.sci.hokudai.ac.jp, shigehiro.nagataki@riken.jp, \\ tamii@rcnp.osaka-u.ac.jp, utsuno.yutaka@jaea.go.jp
}

Photodisintegration is a main process of energy losses of ultrahigh energy cosmic ray (UHECR) nuclei at the highest energies when UHECR nuclei propagate in intergalactic space. Therefore, the model dependence of the photodisintegration is important in the intepretation of experimental data. We calculated photonuclear reactions of 27 stable nuclei with nuclear-density functional theory (DFT) using the random-phase approximation (RPA) and three interaction models. We implemented the results in a public cosmic-ray propagation code CRPropa and simulated cosmicray propagation from extragalactic sources. We present the comparison of the simulated energy spectra between the models.

$37^{\text {th }}$ International Cosmic Ray Conference (ICRC 2021)

July 12 th - 23rd, 2021

Online - Berlin, Germany

\footnotetext{
*Presenter
} 


\section{Introduction}

A transition to heavier elements of ultra high energy cosmic rays (UHECRs) was observed in the $X_{\max }$ above $10^{18.2} \mathrm{eV}$ by Pierre Auger, and the recent results of their $X_{\max }$ can be found in [1]. Therefore, UHECR nuclei propagate in intergalactic space to reach the earth if UHECR nuclei are emitted from some extragalactic sources. The most important interaction of UHECR nuclei in the propagation at the highest energies is photodisintegration with cosmic microwave background (CMB) photons. Especially the photodisintegration via giant dipole resonances (GDRs) is important.

The impact of the model dependence of photonuclear reactions on the observables was simulated in the previous works such as [2,3]. In this work, the random phase approximation (RPA) calculations in density functional theory (DFT) $[4,5]$ were applied to photonuclear reactions in propagation of UHECR nuclei. Then we obtained the impact of the model dependence on the model predictions of energy spectra.

\section{Method}

- We did the RPA calculations of 27 stable nuclei $\left({ }^{12} \mathrm{C},{ }^{13} \mathrm{C},{ }^{14} \mathrm{~N},{ }^{15} \mathrm{~N},{ }^{16} \mathrm{O},{ }^{17} \mathrm{O},{ }^{18} \mathrm{O},{ }^{19} \mathrm{~F},{ }^{20} \mathrm{Ne}\right.$, ${ }^{21} \mathrm{Ne},{ }^{22} \mathrm{Ne},{ }^{23} \mathrm{Na},{ }^{24} \mathrm{Mg},{ }^{25} \mathrm{Mg},{ }^{26} \mathrm{Mg},{ }^{27} \mathrm{Al},{ }^{28} \mathrm{Si},{ }^{32} \mathrm{~S},{ }^{36} \mathrm{Ar}^{40} \mathrm{Ca}{ }^{48} \mathrm{Ti}^{51} \mathrm{~V}^{52} \mathrm{Cr}{ }^{53} \mathrm{Cr}{ }^{54} \mathrm{Cr}{ }^{55} \mathrm{Mn}$ and ${ }^{56} \mathrm{Fe}$ ) for photo nuclear reactions. We used three types of the interaction models [6-8] in the calculations.

- We input E1 strength function of the RPA calculations to TALYS [9], and then we obtained nonelastic cross sections and branching ratios as output of TALYS. This is because the RPA calculations do not provide branching ratios and do not provide photonuclear reactions other than GDRs like quasi-deutron component. Fig. 1 shows an example of calculated nonelastic cross sections of ${ }^{28} \mathrm{Si}$ using different interaction models.

- One dimensional cosmic ray propagation from extragalactic sources to the earth was simulated with CRPropa [10] whose cross sections and branching ratios of 27 nuclei were replaced with calculated ones.

- We compared simulated energy spectrum and $\ln \mathrm{A}$ on the earth with simulated ones using default settings of CRPropa.

\section{Impact on model predictions of cross sections of GDRs}

The peak energies and cross sections of GDRs predicted using RPA calculations are systematically different from CRPropa as shown in Fig. 2 and Fig. 3. The peak energies obtained with RPA calculations especially using $\mathrm{SkM}^{*}$ and SLy4 interaction models tend to be lower and the peak cross sections tend to be higher than other models. The mean free paths are expected to reflect this tendency directly. Fig. 4 shows mean free paths using nonelastic cross sections in Fig. 1. The difference of the peak energies of GDRs shifts the Lorentz factors in this figure, and the difference of the cross sections of GDRs shifts the mean free paths. 


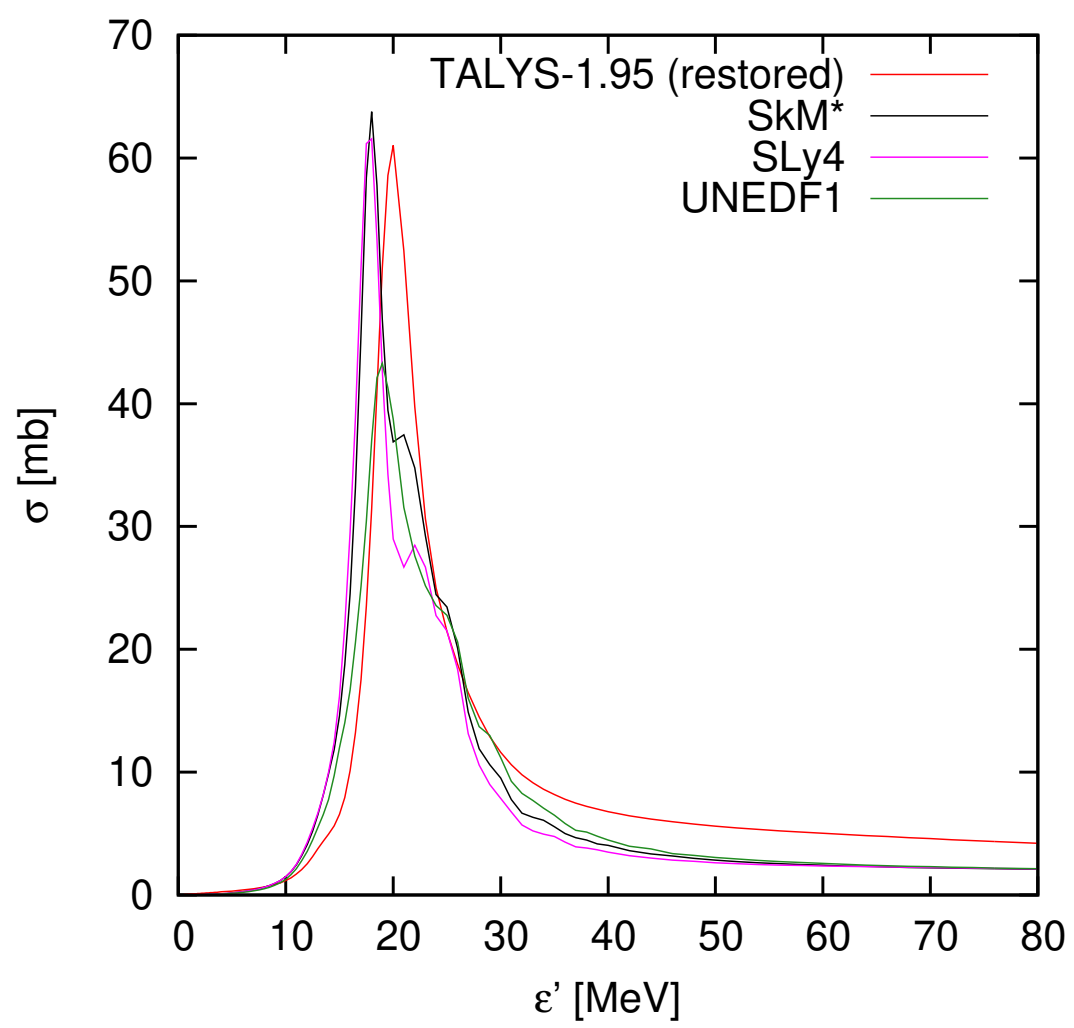

Figure 1: Model predictions of nonelastic cross sections of ${ }^{28} \mathrm{Si} . \epsilon^{\prime}$ means photon energy in the nucleus's rest frame. Red solid line is implemented in CRPropa. Black, pink and forest green line show the predictions using the random phase approximation calculations with different interaction models.

\section{Impact on model predictions of UHECR spectra}

The following astrophysical parameters of extragalactic sources are taken not to contradict the experimental data for the results of CRPropa.

- Injection energy spectrum $d N / d E \propto E^{+0.80}$.

- Broken exponential rigidity dependent cutoff $\log \left(R_{\text {cut }} / \mathrm{eV}\right)=18.20$.

- Evolution of number of sources $(1+z)^{4.2}$ in the comoving unit volume is assumed.

- Energy scale of the data is shifted up by $+12 \%$. This shift is within the systematic uncertainty $14 \%$ of the experimental data [11].

- Relative abundance of five elements are assumed at the source at $10^{18} \mathrm{eV}$. Relative fraction of the H, He, N, Si and Fe nuclei are 0.0, 0.939, 0.052, 0.008 and 0.0002, respectively.

- [13] is used as the extragalactic background light model.

We simulated the propagation with these parameters using different photonuclear reaction models and compared with the experimental data. We fitted only the normalization of the energy 


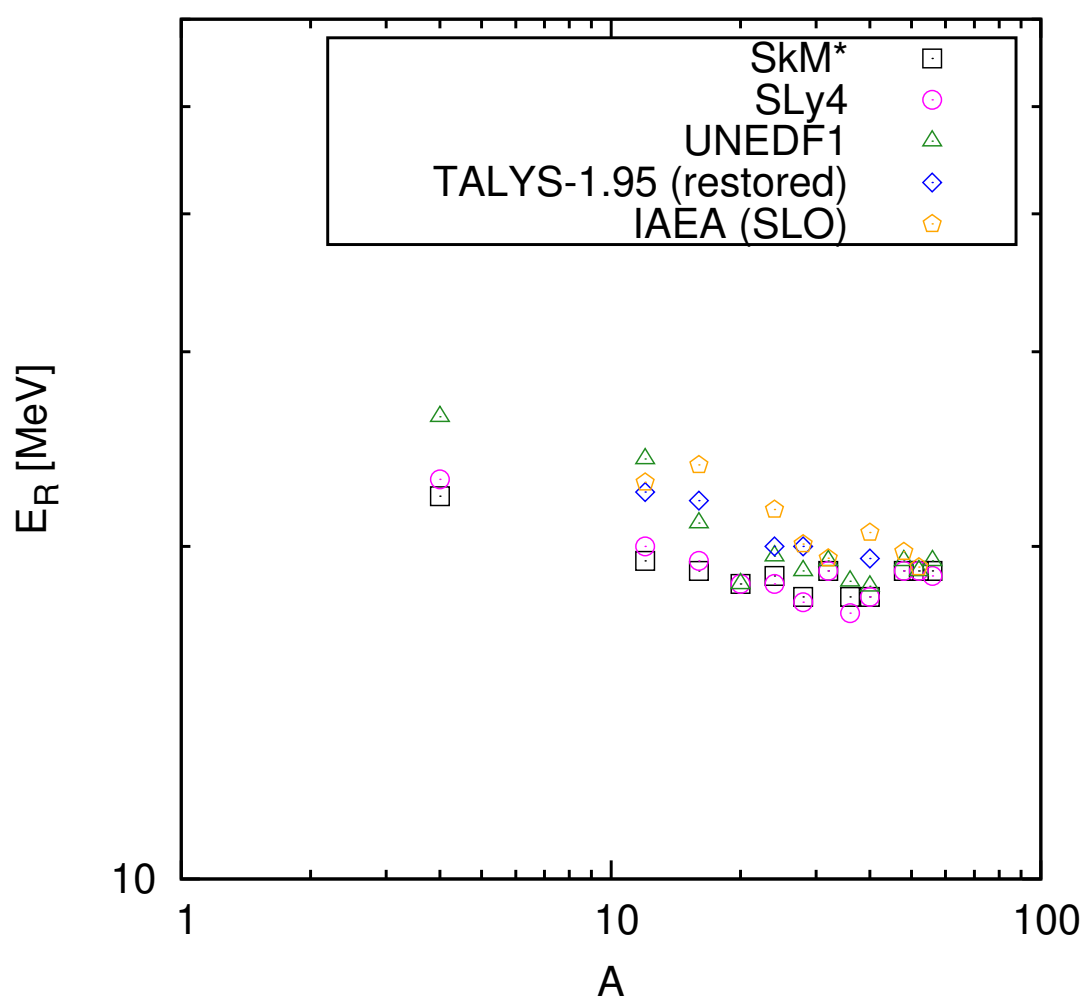

Figure 2: Model predictions of peak energies of giant dipole resonances. The yellow hexagon shows the standard Lorentzian model of the experimental data [12]. The blue diamond shows the cross section included in CRPropa. Black square, pink circle and forest green rectangle show the cross sections obtained with the random phase approximation calculations using different interaction models.

spectrum with $E>10^{18.7} \mathrm{eV}$ to compare different models. Fig. 5 and Fig. 6 show the results of the comparison. We also compared the model predictions of mean $\ln \mathrm{A}$ and $\sigma(\ln \mathrm{A})$ on the earth, and the difference between photonuclear reaction models is much smaller than that of the spectral shape and within the statistical uncertainty of the experimental data.

\section{Summary and Conclusions}

The peak energies and cross sections of GDRs are systematically different from CRPropa as shown in Fig. 2 and Fig. 3, and the difference resulted in the difference of the spectral shape shown in Fig. 5 and Fig. 6. We found that the difference is much larger than the statistical uncertainty of the experimental data. The model predictions of the peak energies and cross sections will be experimentally tested by the Photo-Absorption of Nuclei and Decay Observation for Reactions in Astrophysics (PANDORA) project.

\section{Acknowledgement}

This work is supported by by the Pioneering Program of RIKEN for Evolution of Matter in the Universe (r-EMU). 


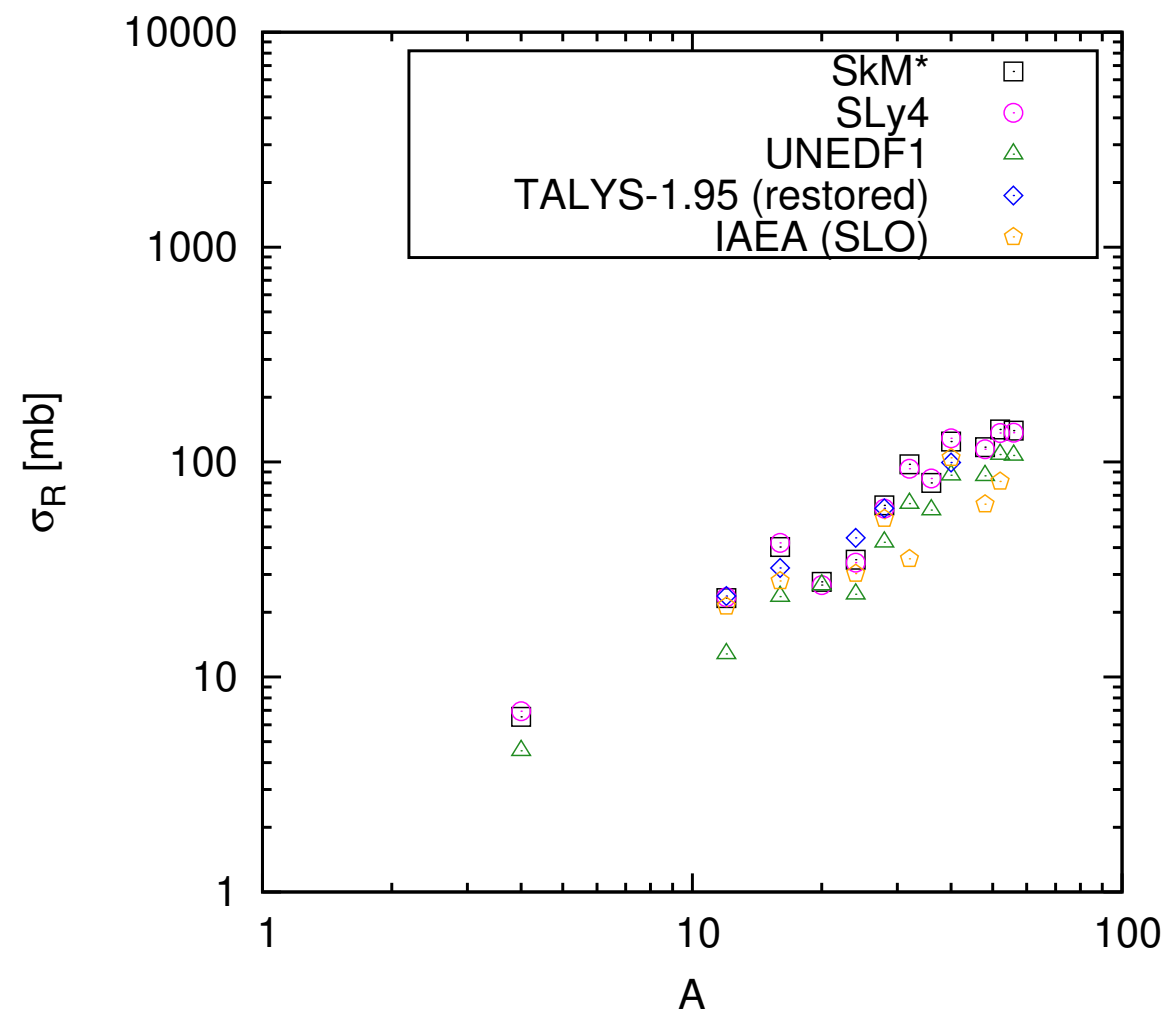

Figure 3: Model predictions of peak cross sections of giant dipole resonances. The meaning of the points is the same as in Fig. 2.

\section{References}

[1] The Pierre Auger Collaboration, arXiv:1909.09073 (2019).

[2] R.A. Batista et al., JCAP 10, 063 (2015).

[3] J. Heinz et al., ApJ, 873, 88 (2019).

[4] T. Inakura et al., Phys. Rev. C 80, 044301 (2009).

[5] T. Inakura et al., Phys. Rev. C 84, 021302(R) (2011).

[6] J. Bartel et al., Nucl. Phys. A 386, 79 (1982).

[7] E. Chanbanat, P. Bonche, P. Haensel, J. Mayer, and R. Schaeffer, Nucl. Phys. A627, 710 (1997).

[8] M. Kortelainen et al., Phys. Rev. C 85, 024304 (2012).

[9] A. J. Koning, S. Hilaire and M.C. Duijvestijn, AIP Conf. Proc. 769, 1154 (2005).

[10] R.A. Batista et al., JCAP 1605, no. 05, 038 (2016); arXiv:1603.07142. 


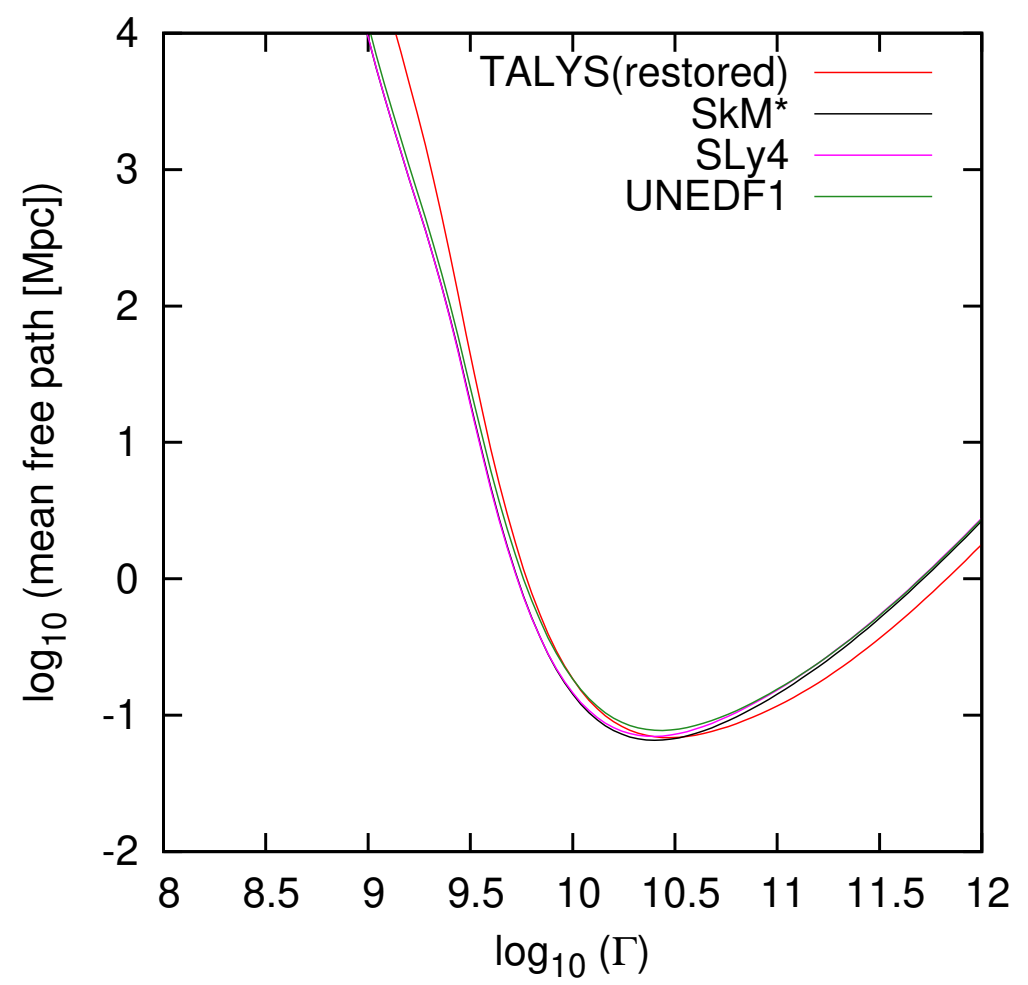

Figure 4: Model predictions of mean free paths using the cross sections of Fig. 1 with cosmic microwave background photons when redshift $z=0 . \Gamma$ is a Lorentz factor of a ${ }^{28} \mathrm{Si}$ nucleus.

[11] V. Verzi, for the Pierre Auger Collaboration, Proc. of 33rd Int. Cosmic Ray Conf., Rio de Janeiro, Brazil (2013) [arXiv:1307.5059].

[12] T. Kawano et al., Nuclear Data Sheets 163, 109-162 (2020).

[13] R.C. Gilmore, R.S. Somerville, J.R. Primack and A. Dominguez, Mon. Not. Roy. Astron. Soc. 4223189 (2012) [arXiv:1104.0671]. 


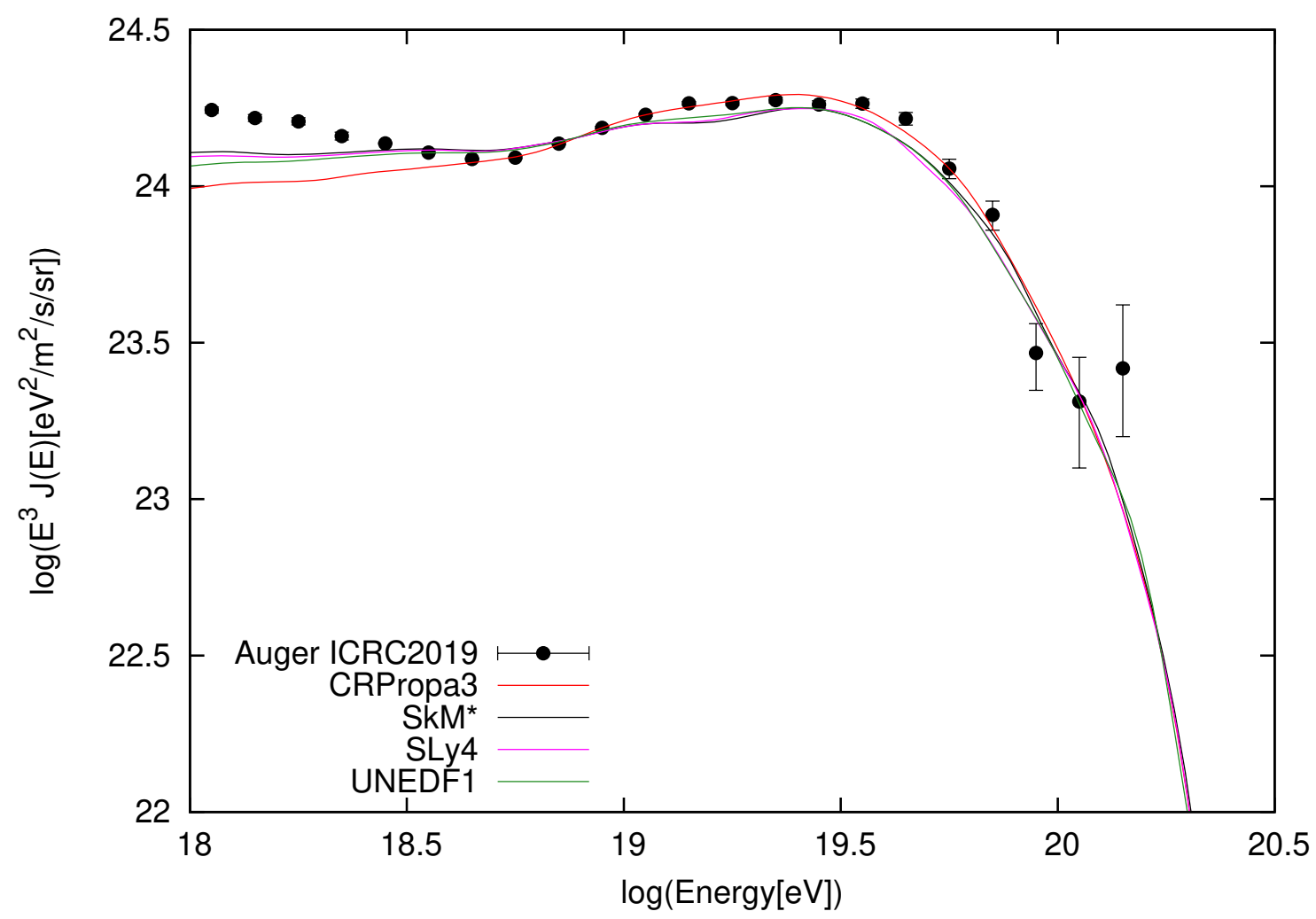

Figure 5: Comparison of simulated energy spectra and the experimental data by Pierre Auger [1]. 15 data points $\mathrm{E}>10^{18.7} \mathrm{eV}$ were used to fit the normalization of simulated energy spectra with the same astrophysical parameters in Section. 4. Black dots are the experimental data. Red solid line is the energy spectrum simulated using CRPropa. Photonuclear reactions of black, pink and forest green solid lines in their simulations of propagation of CRPropa are obtained based on the random phase approximation calculations using different interaction models. $\chi^{2} /$ d.o.f. of the red line, black line, pink line and forest green line are $25.1 / 14,361 / 14,334 / 14$ and 236/14, respectively. 


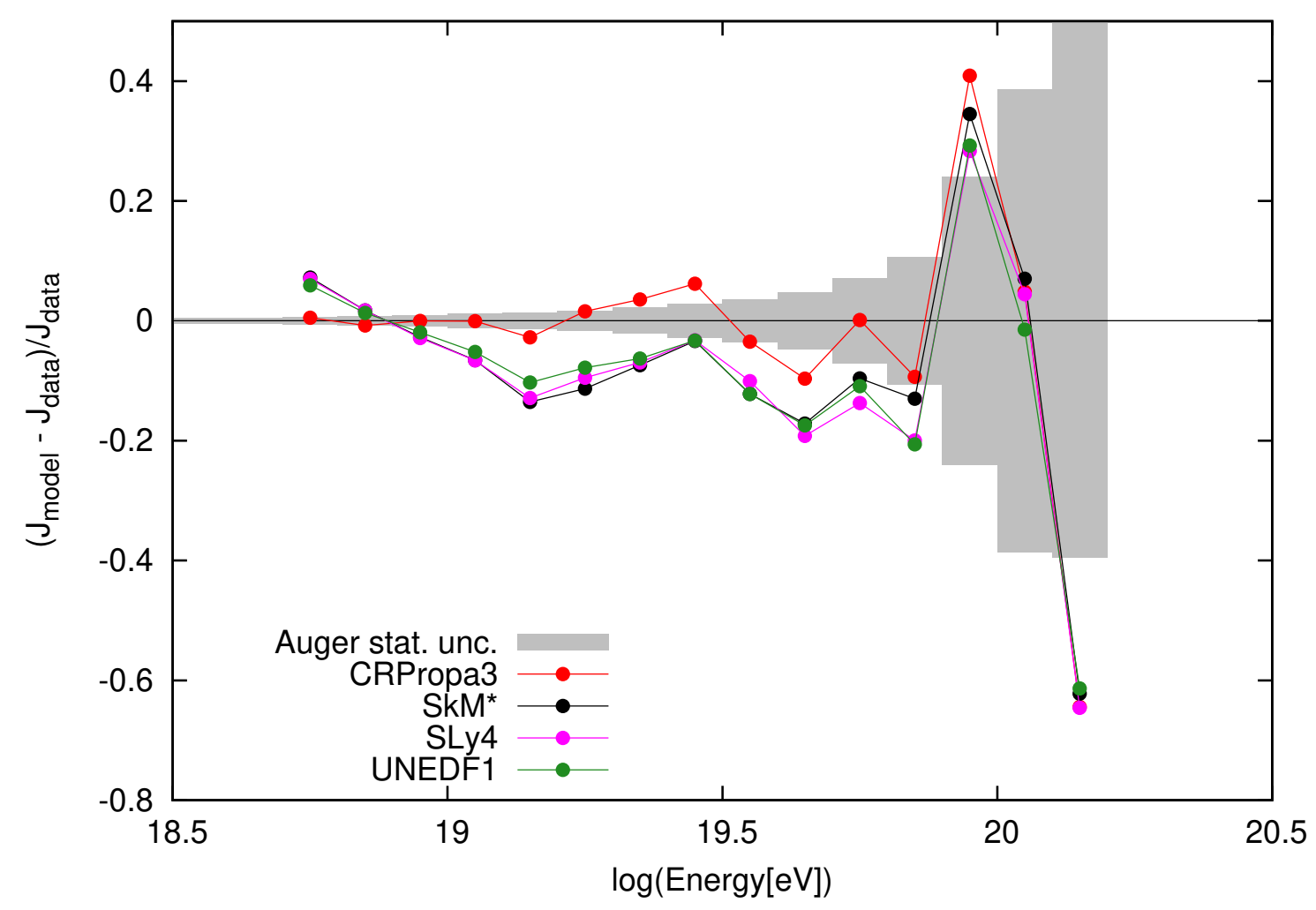

Figure 6: Same results as Fig.5 are plotted here. In this figure, the relative differences of the model predictions of the intensities $\mathbf{J}_{\text {model }}$ from the data $\mathbf{J}_{\text {data }}$ are plotted. The statistical uncertainties of the data are described as hatched region. 